\title{
Il dominio egiziano nelle Cicladi sotto Tolomeo Filopatore.
}

\section{Di Vincenzo Costanzi.}

Nell' ultimo decennio del regno di Tolomeo Filadelfo, la sua flotta fu sconfitta a Kos da Antigono Gonata, come ricaviamo da testimonianze troppo scarse per una perfetta ricostruzione del periodo di cui la battaglia menzionata fu l'epilogo o un episodio, ma sufficienti per congetturare il posto che essa dovette avere nella serie degli avvenimenti, e stabilire una cronologia approssimativa ${ }^{1}$. Una disfatta navale subita dalla flotta egiziana arrebbe dovuto avere gravi conseguenze pel dominio o almeno pel prestigio dell' impero tolemaico nel mare Egeo; tuttavia nell' iscrizione adulitana tra $i$ possedimenti che l'Evergete arrebbe ereditati dal padre vengono enumerate anche le Cicladi, che apparirebbero come il frutto più desiderabile d'un successo navale pel re di Macedonia ${ }^{2}$ ). Senza dubbio il linguaggio apologetico del documento suscita ginstamente la nostra diffidenza; ma se possiamo ammettere in esso esagerazioni e travisamenti, non è concepibile una menzogna che oltre all' essere impudente, sarebbe stata anche ridicola, perché l'Evergete con l'affermazione della sovranità sopra un paese strappato alla propria dominazione non avrebbe fatto altro che commemorare un disastro. Pertanto corrisponde ai dettami d'una critica prudente l'ipotesi che l'impero tolemaico, se potè essere in quell' occasione menomato nelle Cicladi, non per questo venisse del tutto abolito.

Le stesse considerazioni sulle conseguenze della battaglia di Kos valgono per quelle della battaglia d'Andro o, qualora si nutra ancora qualche

1) Per la battaglia di Kos cfr. Athen. p. $209 \mathrm{E}$; Plut. Mor. p. $545 \mathrm{~B} ; 183 \mathrm{D}$; 676 F (?) ; Diog. Laert. IV 6,39. La connessione con la guerra cremonidea, supposta dal Droysen (III 1, p. 240 sg.) e dal Niese (II p. 131 sg.), è inconciliabile col passo di Diogene Laerzio testè citato, dal quale risulta che Atene era in potere d'Antigono. La cronologia (circa 256 a. Cr.) e le combinazioni più probabili sono quelle proposte dal Beloch (III 1 p. 618; III 2 p. 431-6); e se anche fossero giuste le considerazioni di Giulio Augusto Levi (Atti della $R$. Accademia delle Scienze di Torino XXXIX p. 629-632), si scenderebbe solo di qualche anno.

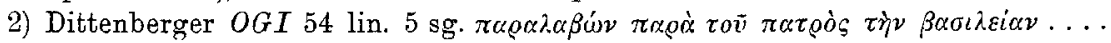
$x \alpha i \lambda \tau \bar{\omega} \nu H v \propto \lambda \dot{\alpha} \delta \omega \nu \nu \dot{\eta} \sigma \omega \nu * \tau \lambda$. 
dubbio circa l'identità di questa con quella presupposta nel cenno di Trogo (Prol. XXVIII), della battaglia che spianò ad Antigono Dosone la via alla conquista totale o parziale della Caria ${ }^{1}$ ). Certamente Amorgo ${ }^{2}$ ) Nisiro ${ }^{3}$ ) e $\mathrm{Kos}^{4}$ ) ad Oriente, Siro ${ }^{5}$ ), forse anche Andro, Paro e Citno ${ }^{6}$ ) ad Oecidente erano sotto il patronato del re di Macedonia. Delo, in eui Antigono Dosone dedicò un monumento commemorativo della battaglia di Sellasia 7), era certo sottratta all' influenza tolemaica. Ma da ciò non consegne che le Cicladi fossero tutte perdute per i Tolomei e fosse subentrata all' egemonia tolemaica la macedonica, come si è recentemente supposto (Delamarre ib. p. 323). Lo stesso linguaggio di Livio nel narrare il ritorno dei Rodii in Asia renderebbe per se stesso molto discutibile questa illazione, perchè egli designa solo Andro, Paro e Citno come stazioni di presidii macedonici (Liv. XXX 115. 8). D'altra parte, se anche ci mancasse ogni indizio di dominio tolemaico nelle isole, si ricarerebbe indirettamente dal fatto che nel Peloponneso ${ }^{8}$ ), a Creta ${ }^{9}$ ), nella (onia ${ }^{10}$ ),

1) Ho enumerate le varie opinioni in Rivista di Filologia XXXVII p. 518-519.

2) Delamarre, Revue de Philologie XXVI [1902] p. 301 sg. specialmente p. $317 \mathrm{sg}$. $S$ ' intende che, dovendo dimostrare la sopravvivenza dell' impero tolemaico nelle acque dell' Egeo, poco importa se alcune isole come Amorgo e Nisiro si possano a rigore chiamare Cicladi. Cfr. Hiller von Gürtringen in IG XII 5 pars altera p. I-II.

3) Dittenberger I² 263 , da cui si rileva che Nisiro era sotto il dominio di Fi-

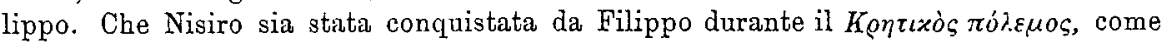
inclinano a credere il Dittenberger e l'Herzog (Klio II p. 328), è molto improbabile, quando si riconosca il Dosone nell' Antigono nominato ripetutamente nelle iscrizioni amorgine riportate dal Delamarre (cfr. n. prec.). L'iscrizione (IG XII 3, p. 17) che l'Herzog (l. c.) vorrebbe riferir alla guerra cretese del 204-201 è dall' Hiller von Gärtringen ( $I G$ n. 103) posta anche per ragioni paleografiche nel 153 av. Cr.

4) Vedi Beloch III 2 p. 463; Sammlung d. Dial. Inschr. 3611; Delamarre ib. p. 314.

5) Delamarre ib. p. 310 .

6) La spiegazione del De Sanctis (Klio IX p. 64.2) che l'Adoís di Plutarco

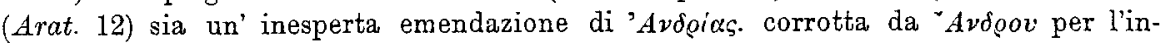
fluenza del seguente $\pi$ oर $\varepsilon_{u} \alpha \varsigma$, mi sembra per ragioni geografiche e paleografiche plausibilissima. Ma da ciò non consegue necessariamente che Andro abbia senza interruzione appartenuto alla Macedonia fin dalla battaglia di Kos o almeno sin dalla liberazione di Sicione (v. sotto), e tanto Andro che Paro e Citno (Liv. XXXI 15) possono essere stati recuperate da Filippo al tempo della guerra rodio-cretese. Per Paro saremmo certamente più informati se l'epigrafe $I G$ XII 5 parte prima n. 125 (p. 37) ci fosse conservata meno lacera.

7) Holleaux in $B C H$ XXXI p. 94 sg. Quando Delo abbia cessato di appartenere al xoıvóv delle Cicladi (dato pure che questo xocvóv continuasse ad esistere pure essendo il dominio di esse frazionato), è superfluo ricercare, per la natura troppo circoscritta della questione che ci occupa. Vedi in ogni modo Holleaux $i b$. p. 114 n. 2; Graindor, Mélanges Kurth p_.4; von Schöffer in Pauly-Wissowa IV 2 p. 4283.

8) $I G$ XII 3 n. $466-7$.

9) Ib. e Strab. p. 472.

10) Beloch III 2 p. 277. Efeso, Samo, Lebedo erano certo tolemaiche alla fine del terzo secolo. Intorno a Mileto si può avanzare quaiche dubbio. 
nella Tracia ${ }^{1}$ ) duravano possessi tolemaici alla morte di Tolomeo Filopatore: onde sarebbe ben strano che proprio nell' arcipelago i Tolomei non fossero riusciti a conservare neanche un avamposto. Tera almeno era tolemaica fino ai tempi del Filometore ${ }^{2}$ ), ed è appena credibile che il suo acquisto datasse dai tempi di lui o dell' Epifane.

Contro questi dati positivi hanno un valore molto problematico le induzioni tratte dall' inerzia della flotta tolemaica contro le depredazioni di Demetrio di Faro ${ }^{3}$ ) e di Dicearco etolo ${ }^{4}$ ). Noi non sappiamo se le scorrerie fossero dirette contro tutte le Cicladi, ma il contegno di Tolomeo e dei suoi luogotenenti è per lo meno tanto problematico quanto quello di Filippo di Macedonia, che, pur avendo dei possedimenti nelle Cicladi, agi di concerto con questi predoni. Certo l'incuria di T'olomen e di Antigono per la sorte di queste isole, fatte segno alle scorrerie dei predoni sopra ricordati, renderebbe a prima vista accettabile l'ipotesi dell' Holleaux, il quale suppone una prostasia dei Rodi nel Mare Egeo ${ }^{5}$ ). Se questa prostasia ebbe luogo, non assunse però il carattere d'una sorranità riconosciuta: almeno non risulta ciò da alcuna attestazione esplicita ed è in manifesta contraddizione con qualche dato delle fonti. Infatti i Rodii ritornando nel 200 dal Pireo in Asia strinsero alleanza con tutte le isole Cicladi, tranne Andro, Paro e Citno tenute dai presidii macedonici "). Ma l'alleanza sarebbe un controsenso, qualora i Rodii esereitassero allora un protettorato formale sulle Cicladi. Ne è supponibile che i Rodii perdessero $\mathrm{i}$ loro domini in seguito alla battaglia di Lade combattuta nel 200 contro Filippo (Pol. b. XVI 14-15) poichè lo stesso travisamento della verità storica compiuto da Zenone e Antistene, scrittori contemporanei, che gabellarono la battaglia di Lade per una vittoria dei Rodii, mostra che l'impero di Filippo non si ampliò per effetto di essa a danno dei

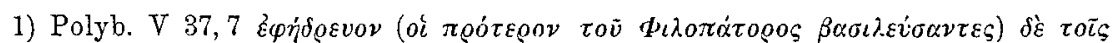

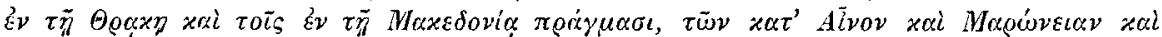

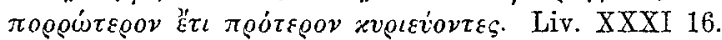

2) Hiller von Gärtringen, Festschrift zu Otto Hirschfeld's LX. Geburtstage p. 87-99, spec. 93-99; Thera I 167 sg.; Beloch III 2 p. 282. 3; Graindor, Mélanges Kurth p. 12 n. 1.

3) Delamarre o. c. p. 323; Homolle BCH XXXI p. 112. - Polyb. V. 35.11 xai

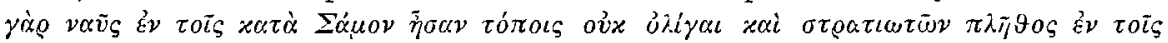

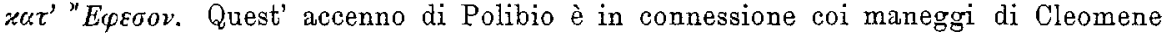
alla corte di Tolomeo Filopatore, e alla politica dei suoi consiglieri, specialmente

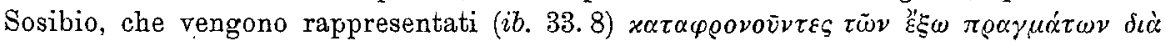

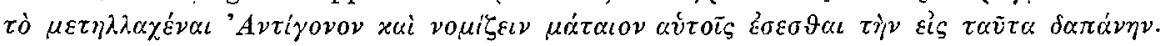

4) Holleaux ib.; Polyb. XVIII 54; Diod. XXVIII 1.

5) Delamarre $i b .324$; Holleaux $i b .114$; Hiller von Gärtringen $I G$ XIr 5 p. $2 \mathrm{a}$ p. XVIIT.

6) Liv. XXXI 15. 8: Rhodii praeter Ciam ab Aegina, inde per insulas Rhodum navigarunt omnibus praeter Andrum Parumque et Cythnum, quae praesidiis Macedonum tenebantur, in societatem acceptis. 
Rodii. Inoltre se Filippo avesse allora strappato ai Rodii le Cieladi, Polibio non si sarebbe limitato, per confutare i prelodati storici, solo a ricordare l'omaggio di Mileto (Polyb. XV 15, 5-8) a Hilippo, ma arrebbe senz' altro rilevato lo strappo d'una parte così cospicua di territorio dall' impero Rodio. Invero senza supporre un' egemonia dei Rodii nelle Cicladi, si comprende come essi per proteggere i loro commerci intraprendessero la difesa delle isole minacciate dalle incursioni, e tra queste quelle soggette all' Egitto si rivolgessero ai Rodii, visto che dia Tolomeo non potevano sperare soccorso ${ }^{1}$ ).

Le ragioni di questa condotta passiva del re d'Egitto vanno cercate nelle condizioni interne del paese. Non occorre ricordare che all' indomani della battaglia di Rafia (217 a. Cr.) incominciò la reazione dell' elemento indigeno contro il greco (Pol. b. V 107,1) e che queste discordie intestine si prolungarono per un buon tratto del regno di Tolomeo Epifane ${ }^{2}$ ). Altri stati hanno trovato l'energia di condurre imprese militari all' estero mentre comprimevano i movimenti rivoluzionari all' interno, ma non era l'Egitto sotto il Filopatore capace di questa forza di resistenza, anche ammesso, anzi forse appunto per questo, che non fosse tutta colpa del monarca la rinuncia ad ogni velleità imperialistica (Polyb. V 34, 8-9). Pertanto, non potendo difendere $\mathrm{i}$ domini insulari che gli erano ancora rimasti, era costretto a tollerare che gli abitanti di questi provvedessero ai casi propri, invocando l'intervento di uno stato interessato a difendere i territori marittimi dalle aggressioni di corsari. Infatti per contrastare con le armi ai Rodi il diritto di stringere trattati con staterelli posti nominalmente sotto la sua sudditanza si sarebbe richiesta quella preparazione e decisione alla guerra necessaria per propulsare le aggressioni piratesche. Ma se è naturale e spiegabile la connivenza dell' Egitto a quest' azione dei Rodii, con i quali non aveva motivi di rivalitì, non sarebbe stato in niun modo da aspettarsi che il sovrano d'Egitto si potesse rassegnare senza reagire ad un' usurpazione manifesta dei suoi territori da parte

1) Non si può vedere l'attestazione d'una sovranità dei Rodii nelle Cicladi in

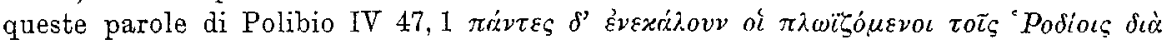

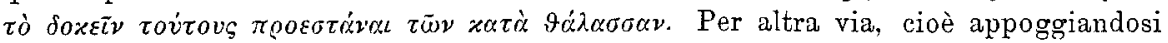
a un passo di Polibio (V 105,6-7) giunge a negare l'egemonia rodia sulle Cicladi il Graindor (ibid. p. 11-12). _Kedi anche Roussel BCH XXXI p. 360, ed Herzog, Klio II p. 333.

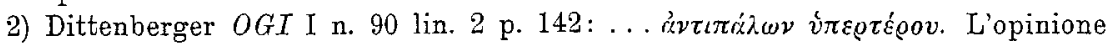
del Mahaffy (The empire of the Ptolemies p. $273=$ History of Egypte p. 140), seguita dal Niese (II p. 406), che connette con queste ribellioni la notizia diodorea (III 6) sulla defezione d'Ergamene da Tolomeo Filadelfo non resiste per nulla alla critica. Poichè, se anche si ammette la possibilità d'un errore di Diodoro, che abbia scambiato il secondo col quarto Tolomeo, o meglio d'una corruttela nel testo di Diodoro

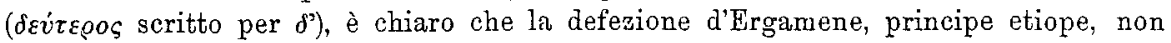
ha nulla a vedere con la riscossa d'un popolo assoggettato. Cfr. Beloch (III 2 p. 286) che tacitamente repudia quest' opinione. 
del re di Macedonia, col quale esisteva un tradizionale antagonismo; e quindi l'anessione delle Cicladi e delle coste dell' Egeo doveano far parte del programma di conquista armata per cui Filippo si alleò con Antioco.

Che Filippo dovesse impadronirsi delle isole e del litorale dell' Egeo sottoposto a Tolomeo, è attestato esplicitamente da Polibio (III 2,8);

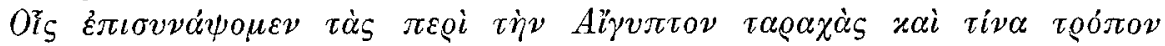

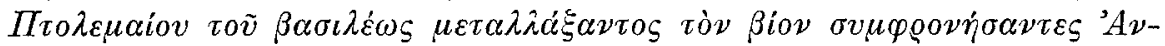

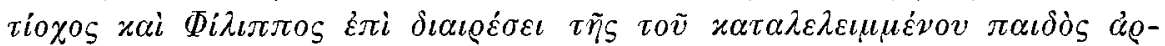

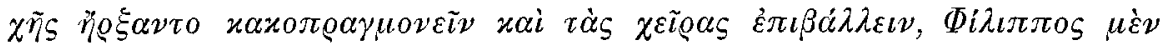

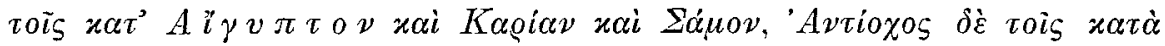

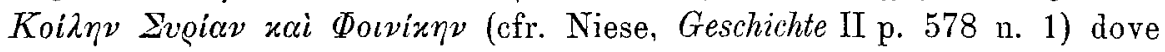

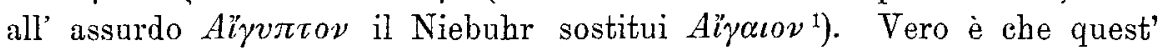
emendazione è stata ritenuta falsa perchè in disaccordo con la cronologia delle guerre di Filippo in Asia negli anni $202-201^{2}$ ), e giudicata d'una grecità sospetta. Ma l'Holleaux il quale vede nelle imprese significate

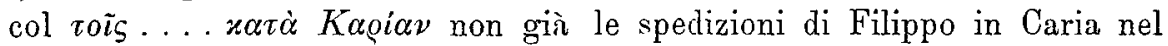
201, bensi le incursioni dei satelliti di Filippo contro Jaso compinte nel 202, quasi contemporaneamente al colpo di mano su Cio, corregge l'Ai-

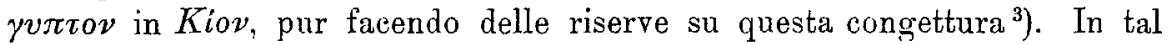
modo gli sembra d'avere eliminata l'unica testimonianza antorevole per l'esistenza d'un residuo di dominio tolemaico nell' arcipelago. Senonchè la correzione dell' Holleaux non solo è incerta, ma secondo il mio avviso inaccettabile. Egli primieramente esige da Polibio un ordine cronologico che nessun scrittore si è mai imposto in un cenno anticipativo, dove formula sommariamente il programma della materia da trattare ${ }^{4}$ ); in secondo luogo la presa di Cio non poteva far parte d'un piano di spartizione dei domini tolemaici tra Filippo e Antioco, poichè Cio non ha mai appartenuto all' Egitto, o almeno non gli apparteneva nell' ultimo decennio del terzo secolo, ma faceva parte della lega etolica ${ }^{5}$ ). Finalmente non sono

1) Abhandlungen der Berliner Akademie 1820.1 p. 106. Vedi le edizioni dell' Hultsch e del Büttner-Wobst.

2) Revue des Études Grecques XII 1899 p. 20. 37, dove l'Holleaux illustra tre iscrizioni rodie contenenti la deliberazione di soccorrere i Jasii contro le incursioni di Olimpico e Podilo, satelliti di Filippo.

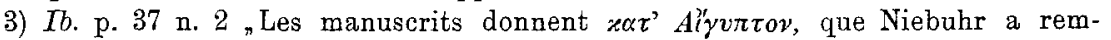
placé par $x \alpha \tau^{\prime} A^{\prime \prime}{ }^{\prime} \alpha \iota t o v$. Cette conjecture a été unanimement accepté par tous les éditeurs plus récents: mais l'expression $x a \tau^{\prime} A^{\prime \prime} y_{\alpha \iota o v}$, pour désigner les îles de la mer Égée, me semble tout à fait insolite; et d'autre part, il serait bien étrange que Polybe n'eût pas rappelé d'un mot les entreprises de Philippe contre les cités de la Propontide : c'est pourquoi que je propose, d'ailleurs sous reserves, la correction $\varkappa \alpha \tau[\dot{\alpha}]$ Kiov "(cioè KATAKION corotto in KATAITYTTTON). Vedi ancora BCH ib. p. 111 n. 2" mais cette conjecture n'est guère acceptable, étant d'une grécité douteuse".

4) Cic. De imper. Cn. Pomp. 6,14; Liv. IX 19,14. In ambedue i luoghi la guerra dei Romani con Antioco è nominata prima di quella con Filippo.

5) Polyb. XV 21,3-8; XVIII 3,12; Liv. XXXII 33, 16; Niese II p. 582.

Kli o, Beiträge zur alten Geschichte XI 3. 
giustificati i dubbi contro la grecità dell' espressione $\left.\varkappa \alpha \tau^{\prime} A i^{\prime} \gamma \alpha \iota \nu^{1}\right)$, e, riconoscinta la legittimità di quest' uso, non vi è ragione di rinunclare al

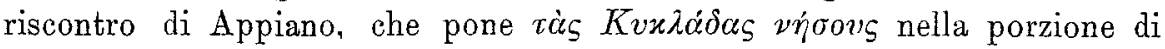
preda spettante a Filippo nella divisione del regno tolemaico tra lui e Antioco, solo perchè il lnogo d'Appiano rigurgita d'errori ${ }^{2}$ ).

Non è adunque temerario supporre che gli effetti della battaglia d'Andro venissero neutralizzati prima dalle complicazioni per cui Antigono Dosone si dovette rassegnare a cedere agli Etoli l'Acaia Ftiotide, la Tessaliotide e l'Estieotide ${ }^{3}$ ), poscia dalla guerra cleomenica, della quale protittava Tolomeo per ricuperare qualche perduto territorio nell' Egeo. La guerra sociale sotto Filippo e forse la guerra successiva con gli Etoli distrassero l'attenzione del giovane re da ogni mira a rignadagnare la posizione che la vittoria navale d'Andro avea pel momento creato alla Macedonia ${ }^{4}$ ). I Rodii che avevano vitali interessi da tutelare profittarono degli imbarazzi del re di Macedonia e dell' inerzia di Tolomeo Filopatore e per assumere un patronato nel mare Egeo, ma a un protettorato formale non pervennero che dopo la seconda guerra macedonica.

1) Nell' espressione $x \alpha \tau^{\prime} A^{\prime}{ }^{\prime} y \measuredangle \iota l o v$ veggo una metonimia nè inusitata nè inelegante. e la coordinazione di $\tau o \bar{\iota} s$ z $\alpha \tau^{\prime} A^{\prime \prime} y \alpha \omega \nu$ con gli altri dre membri indicanti senz' altro i luoghi, meta delle ambizioni di Filippo, è tanto giustificata come la

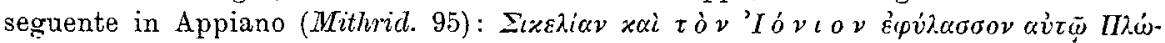

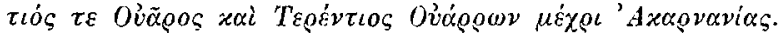

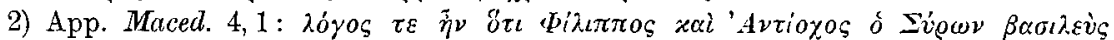

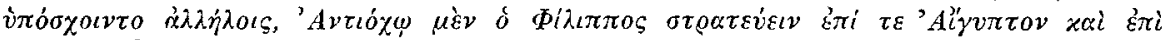

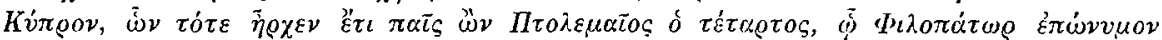

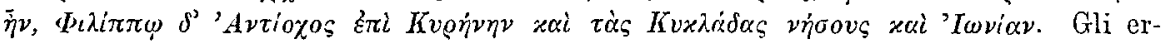
rori che si notano in questo luogo provano solo l'imperizia del compilatore; ma non è questo un motivo per condannare in blocco la testimonianza, come fa l'Holleaux ( $b$. p. 112 n.). Con questo criterio dovremmo rinunciare a valerci non solo di Appiano, ma anche di Plutarco e di Diodoro. Appiano confonde il Filopatore con l'Epifane, e si spiega: nella sua fonte trovava nominato soltanto Tolomeo, ed egli ha supplito a memoria l'epiteto. Appiano inoltre ha, per equivoco o per guasta lezione

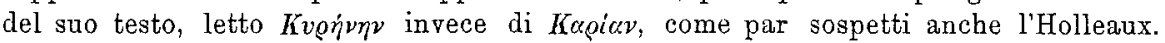

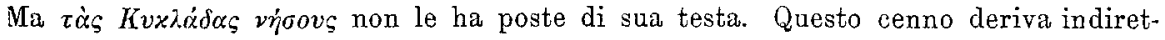
tamente da Polibio.

3) Si potrà discutere sulle modalità, ma la tesi del Beloch (III 2 p. 340 sg.) che queste province fossero incorporate alla lega etolica nei primi anni del regno di Antigono Dosone, è senza dubbio giusta. Inoltre la ribellione dei Tessali (Trog. Prol. XXVIII, Iust. XXVIII 3,14) mostra come non fosse ancor molto salda l'egemonia della Macedonia nei paesi sottomessi o legati con qualche patto federale. Si comprende perciò che non fosse facile conservare i dominii marittimi.

4) La questione se Antigono Dosone o Filippo avesse trascurato l'incremento della marina, è fino a un certo punto secondaria. Del resto dalla lettura dei luoghi di Polibio $(\mathrm{V} 2,4,7,11)$ non si ricava punto che la flotta macedonica fosse stremata del tutto, come ritiene l'Holleaux (ib. p. 107 n. 3). Si comprende come, dovendo lottare con un popolo di pirati come gli Etoli si dovessero raddoppiare le cure e le esercitazioni. Inoltre, dal momento che gli Achei erano alleati dei Macedoni, è chiaro che si cercava di ottenere con la consociazione il massimo delle forze. 
A p p e n dice. - Il manoscritto del presente laroro era stato consegnato da cirea un anno alla direzione del periodico Klio, quando venne alla luce (o almeno venne a mia conoscenza) l'opuscolo Der Bund der Nesioten del dottor W erner König. Anch' egli ritiene (p. 18-31; specialmente p. 28-30) che il dominio egiziano siasi conservato nelle $\mathrm{Ci}$ cladi durante il regno di Tolomeo Filopatore, giungendo spesso per altri via alle mia conclusioni, e consente con me anche nella difesa dell' emendazione proposta dal Niebuhr a Polyb. III 2,8, contro i dubbi sollevati dall' Holleaux riguardo alla grecità della locuzione $x \alpha \tau$ ' Aïraıov. Però a p. 30 n. 7 ha citato per svista, a confoito della congettura niebuhriana, il III 2,8 che è appunto il luogo controverso, e il XVI 34,1 $\delta \imath^{\prime}$ Airaiov

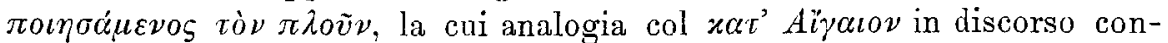
siste solo nella mancanza dell' articolo. Ma non credo che per questa l'Holleaux abbia messo in dubbio la grecitil della locuzione, bensi per l'uso

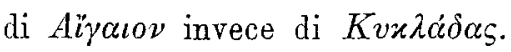

Pisa, febbraio 1911. 